weeks 16, 32 and 36. Type I Interferon (IFN) induced gene signature was determined using a previously validated four gene interferon score (IFI27, IFI44, IFI44L, RSAD2) using quantitative PCR.

Results No local or systemic allergic reactions occurred following Omalizumab injections. Of the 52 adverse events (AEs), 49 were reported as mild while 3 met the criteria for severe AEs. SLEDAI $2 \mathrm{~K}$ scores improved significantly in the Omalizumab group at week $16(p=0.038)$, as well as during the open label phase in subjects initially receiving placebo $(p=0.020)$. No worsening in BILAG scores or PGA were detected. A total of $26 \%(4 / 15)$ patients achieved SRI4 response criteria with Omalizumab. IFN gene signature improved in subjects treated with Omalizumab $(p=0.11)$, especially in subjects with high baseline IFN scores $(\mathrm{p}=0.052)$.

Conclusions Omalizumab in subjects with SLE is well tolerated and associated with improvement in disease activity potentially linked, at least in part, to modulation of type-I IFN responses. Larger randomized clinical trials are required to assess the role of this drug in patients with SLE.

Trial registration

Clinical Trials registration number NCT01716312

\section{Environmental Factors}

\section{EF-01 SOCIOECONOMIC DETERMINANTS AND SLE SEVERITY IN BLACK PATIENTS IN BARBADOS}

Cindy Flower*. University of the West Indies, Cave Hill campus, Barbados

\subsection{6/lupus-2018-Ism.81}

Background SLE patients of lower socioeconomic position (SEP) exhibit increased morbidity and mortality but less clear is whether the disease incidence is increased in the setting of poverty and whether a more severe disease phenotype develops in poorer patients. Crucial etiologic factors for SLE may predominate in genetically-predisposed individuals of lower SEP. Lack of running water at age 12 and frequent childhood infections- both of which are associated with poverty, have been implicated in some groups. In our cohort of black patients in Barbados, we sought to determine by comparison with the general population if SLE clusters in persons of lower SEP and if poorer patients had a more severe disease phenotype.

Methods Patients from the Barbados National Lupus Registry (universal access to care) were included if they had a clinical diagnosis of SLE made by a rheumatologist in the 7 year period 2008-2014.

The clinical status was assessed at the end of 2017 ensuring all patients had at least 3 years of follow up - the period of time within which most severe SLE organ involvement develops. Private health insurance coverage was used a proxy for SEP. Patients were divided into 2 groups based on the presence or absence of health insurance at disease diagnosis. We investigated the effect of SEP on major organ involvement (yes/no), SLE nephritis (yes/no), neuropsychiatric disease (yes/ no), and vital status in December 2017 (alive, alive with chronic kidney disease, died).

Results In a subset of 135 patients with SLE $(F=126), 29$ (21\%) had private healthcare insurance at diagnosis (median age 32), the remaining 106 (79\%) did not (median age 35).
This compares to a national prevalence of private healthcare of $24 \%$ (95\% CI $21 \%$ to $26 \%$ ). The association between health insurance status and each of the four complications/outcomes is presented in table 1 . After adjusting for disease duration, patients without private health insurance at diagnosis had more complicated SLE and worse SLE outcomes (Odds Ratios $>1$ ) in all cases. For two of these clinical complications (major organ involvement and SLE nephritis) the effects were borderline statistically significant at the 5\% level.

Conclusions Socioeconomic determinants appear to affect the severity of SLE in Barbados with poorer patients having a more severe disease phenotype.

The high morbidity and mortality documented in the international literature in the setting of poverty may be due to a combination of social issues and the presence of more aggressive disease from the outset.

\begin{tabular}{|c|c|c|c|}
\hline Outcome & Odds ratio & $95 \% \mathrm{Cl}$ & $P$ value \\
\hline Major organ involvement $\dagger$ & 2.69 & $1.00-7.26$ & 0.05 \\
\hline Lupus nephritis $\dagger$ & 3.24 & $1.01-10.37$ & 0.05 \\
\hline Neuropsychiatric lupus $\dagger$ & 4.40 & $0.55-35.16$ & 0.16 \\
\hline Vital status & 3.83 & $0.84-17.45$ & 0.08 \\
\hline
\end{tabular}

\section{EF-02 LONGITUDINAL STUDY OF LONG-TERM POVERTY AND PERSISTENT DEPRESSIVE SYMPTOMS IN SLE}

${ }^{1}$ Ed Yelin*, ${ }^{1}$ Jinoos Yazdany, ${ }^{1}$ Laura Trupin, ${ }^{2}$ Natalie McCormick, ${ }^{1}$ Patricia Katz. ${ }^{1}$ University of California, San Francisco, San Francisco, USA; ${ }^{2}$ University of British Columbia, Vancouver and Arthritis Research Centre of Canada

\subsection{6/lupus-2018-Ism.82}

Background A prior study found that persons with SLE in longterm poverty have greater accumulation of disease damage over 6 years than those exiting poverty or never in poverty. ${ }^{1}$ The present study evaluates the effect of long-term poverty status on depressive symptoms over the same duration of time.

Methods Data are from the UCSF Lupus Outcomes Study in which persons with SLE were recruited in 2003 throughout the U.S. and interviewed annually through 2015. In each year we characterized respondents' poverty status based on household income and family size and administered the CESD measure of depressive symptoms, defining a high level of depressive symptoms using a validated SLE-specific cutpoint $(\geq 24)$ associated with a formal diagnosis of depression. ${ }^{2}$ Prevalent persistent depression was defined as having high levels of depressive symptoms for $\geq 3$ years between 2009 and 2015. Incident persistent depression used the same criteria, measured only among those who had low levels of depressive symptoms between 2006 and 2009. Logistic regression was used to estimate the impact of being poor in every year from 2003-2009, permanently leaving poverty by 2009, or never being poor on prevalent and incident persistent depression, with and without adjustment for gender, age, marital status, race/ethnicity, education, disease duration, extent of accumulated damage by 2009 using the Brief Index of Lupus Damage, ${ }^{3}$ smoking status, and BMI. 